\title{
Monodispersed, Highly Interactive Facet (111)-Oriented Pd Nanograins by ALD onto Free-Standing and Flexible Electrospun Polymeric Nanofibrous Webs for Catalytic Application
}

\author{
Kugalur Shanmugam Ranjith, Asli Celebioglu, Hamit Eren, Necmi Biyikli, \\ and Tamer Uyar**
}

An atomic layer deposition (ALD) of monodispersed palladium (Pd) nanograins $(\approx 2 \mathrm{~nm}$ ) onto electrospun polymeric nanofibers (NF) is presented. By ALD, monodispersed Pd nanograins with (111) exposed facets are decorated on the surface of the free-standing flexible nanofibrous webs (NW). The Pd nanograin-decorated free-standing NW exhibit catalytic reduction of 4-nitrophenol to 4-aminophenol. Even under low loading capacity $\left(\approx 20 \mu \mathrm{g} \mathrm{mg}^{-1}\right)$, Pd nanograins manifest effective catalytic performance which can be referred to direct exposure of Pd single crystalline highly interactive (111) plains with high surface area on the NW. The Pd nanograins and the interactive sites along with the high surface area NW yield effective catalytic reduction of 4-nitrophenol to 4-aminophenol with the catalytic reduction rate of $0.0531 \mathrm{~min}^{-1}$. Pd nanograins display thermally tunable effective catalytic reduction properties with activation energy $\left(E_{\mathrm{a}}\right)$ of $1.705 \mathrm{~J} \mathrm{~mol}{ }^{-1}$ on varying the reaction temperature from 12 to $42{ }^{\circ} \mathrm{C}$. Moreover, $\mathrm{Pd}$ nanograin-decorated NW are exhibited the effective reusable behavior with stable structural integrity even after repeated catalytic reactions. The approach of this study opens up synthesis and surface decoration of metal nanostructures onto NF through ALD with controlled size and facet orientation for designing reusable and free-standing flexible catalytic nanofibrous materials.

\section{Introduction}

Hydrogenation of industrial toxic pollutants such as aromatic nitro components (4-nitrophenol, 4-NP) into commercially important 4-aminophenol (4-AP) is a topic of hot interest for

Dr. K. S. Ranjith, Dr. A. Celebioglu, H. Eren, Prof. T. Uyar Institute of Materials Science and Nanotechnology and UNAM-National Nanotechnology Research Center Bilkent University

Ankara 06800, Turkey

E-mail: tamer@unam.bilkent.edu.tr

Prof. N. Biyikli

Electrical and Computer Engineering

University of Connecticut

Storrs, CT 06269-4157, USA

The ORCID identification number(s) for the author(s) of this article can be found under https://doi.org/10.1002/admi.201700640.

DOI: 10.1002/admi.201700640 industrial applications. ${ }^{[1,2]}$ Initially, these toxic nitro components were converted through the electro-fenton process, photocatalytic degradation, electrocoagulation, hydrogenation, etc. ${ }^{[2-6]}$ In recent years, metal nanoparticles have been subjected intensively as a catalyst based on its functional properties when it down to nanoscale. ${ }^{[7]}$ Catalytic activity through hydrogenation of excessive $\mathrm{NaBH}_{4}$ with metal-based nanostructures exhibited efficient transformation of toxic pollutants with commendable economical process by reduction of 4-NP to 4-AP. ${ }^{[8]}$ Utilizing noble transition metal ions such as gold $(\mathrm{Au})$, silver $(\mathrm{Ag})$, palladium $(\mathrm{Pd})$, and platinum (Pt) as an efficient catalyst has proven to be successful in the hydrogenation process. ${ }^{[9]}$ Among noble metal nanocatalysts, Pd nanostructures have been significantly utilized for the reduction of hydrogenation, due to their superior activity and higher stability with promising electronic and optical functionality and reusability than other noble metal nanostructures. ${ }^{[10,11]}$ Due to the higher price and scarce availability of noble metals, some research is focused on the bimetallic functionality by combining the noble metal ions with transition metal ions to improve its functionality ${ }^{[12]}$ But, before necessary steps are taken on this juncture, it is to be noted that due to their poor thermodynamical stability, noble metal ions at the higher active centers minimize their total surface energy by aggregation process, thereby minimizing their catalytic functionality. ${ }^{[13]}$ To avoid aggregation and bring forth efficient catalytic behavior with lower quantity of the metal nanostructures, a process was initiated by combining the role of surfactants and stabilizers. ${ }^{[14]}$ But these stabilizers which cap the nanostructures could prevent the aggregation, but sometimes adversely affect the catalytic activity, which is not suitable for the scale-up process.

The size and the shape of the metal nanostructures are crucial factors in the sensitive reactions, and the facet arrangement will play a role in improving the sensitivity and stability of the Pd metal nanoparticles during the catalytic process. ${ }^{[15,16]}$ 
$\mathrm{Xu}$ et al. ${ }^{[17]}$ have revealed that exposure of the (111) facets of the $\mathrm{Pd}$ nanocatalyst plays a promising role in improving the $\mathrm{CO}$ oxidation process with the long-lived catalytic activity even for lower loaded Pd nanostructures. Johnson et al. ${ }^{[6]}$ have predicted that the size and support-based design on the formation of Pd base dendrimers improved the catalytic activity of the nanostructures. Over the different morphology of noble metal nanostructures, the exposition of (111) facets exhibited higher catalytic activity than the (100) facet-oriented metal nanostructures. ${ }^{[18-20]}$ In electro-oxidation process, single (100) facet orientation significantly reveals the better performance on the Pd- and Pd-Pt-based bimetallic nanostructures toward its catalytic behavior. ${ }^{[21,22]}$ Additionally, providing substrate or support for loading or growing the noble metal ions exhibits efficient catalytic performance by selective growth orientation and by avoiding the agglomeration of metal nanostructures. The solution-based chemical reduction process facilitates the synthesis of different morphology of metal nanostructures with different facet-oriented planes, but it does not favor the interaction of metal ions with the supportive substrate which affects the durable nature of the catalyst medium.

In previous investigations, mostly high-surface-area porous materials such as charcoal, silica, alumina, zeolite, carbon fiber, and carbon nanotubes were used as support material for the catalyst, and they further utilized in many functional applications. ${ }^{[23]}$ But after the metal decoration, these supports have had some disadvantages such as difficulty to handle in the catalytic environment for reusability. Based on this consideration, for instance, flexible electrospun polymeric NF and their NW were used as a substrate that has a 1D network with controllable diameter and composition with industrial scale productivity. Unique structural features along with high porosity and high surface area make electrospun $\mathrm{NF} / \mathrm{NW}$ as one of the promising free-standing and flexible substrate for metal nanoparticles decoration. ${ }^{[24,25]}$ Hence, the electrospun NF/NW can work as a facile platform for the metal decoration and avoid aggregation by surface interactivity.

Precisely controlling the facet orientation of the metallic nanostructures on the supportive substrate with the controlled morphology is highly complicated in the chemical reduction process without using surfactant or capping agents. Hence, ALD can be employed for the controlled growth of metal nanostructures at the atomic level. ALD is a relatively low-temperature deposition process, so temperature-sensitive substrates such as polymeric materials can be decorated by metallic nanoparticles by ALD. By controlling the ALD cycles, it is possible to control the facets and the size distribution and the loading of metal nanostructures on the substrates. In this work, through ALD, ultra-small, monodispersed, long-time durable, Pd nanograins were deposited over the flexible substrate of electrospun polymeric NW. Hydrogenation-based catalytic reaction was initiated over Pd nanograin-decorated NW respectively with the loading of $\mathrm{Pd}$ in the catalytic activity. Also, the influence of temperature during the catalytic reduction and the catalytic and structural stability after consecutive cycles were investigated. This study reveals the effective approach by ALD in order to fabricate the surface-decorated metal nanostructures onto flexible and free-standing polymeric NW for the efficient catalytic applications.

\section{Result and Discussion}

We fabricated ultra-low dimensional, (111) facet-oriented Pd nanograins $(<2 \mathrm{~nm})$ on the polymeric NF for the hydrogenation-based catalytic application. As illustrating in the schematic diagram, we have firstly obtained bead-free and uniform electrospun NF from three different types of polymeric materials (Nylon and Polyacrylonitrile (PAN) and Polysulfone (PSU)) by using electrospinning technique; afterward, Pd nanograins were decorated via ALD on these polymeric NW (Figure 1). The scanning electron microscope (SEM) images clearly evidence the homogeneous morphology of pristine Nylon NF and PAN NF which were produced through electrospinning process (Figure 2a,c). The electrospun Nylon NF and PAN NF were having the fiber diameter of $60 \pm 10 \mathrm{~nm}$ and $400 \pm 10 \mathrm{~nm}$, respectively. On inducing these NW as substrate for Pd decoration during ALD process, it was elucidated that these Nylon NW and PAN NW were structurally quite stable after Pd nanograin decoration. When compared to the pristine Nylon NF and PAN $\mathrm{NF}$, there were some notable changes on the surface of the NF after ALD process due to the surface decoration of NF with $\mathrm{Pd}$ nanograins. Figure 2e-f and Figure S1 (Supporting Information) show that, after the ALD deposition of Pd, polymeric NW are still flexible with free-standing nature. After the ALD deposition process, the pristine white color of the Nylon and PAN NW were changed to gray and yellow, respectively. For the electrospun PSU NF sample, the morphology of the PSU fibers could not be protected, and after ALD process, it was observed that the fibers were somewhat fused to each other possibly due to the formalin environment and deposition temperature of ALD (Figure S2, Supporting Information). Therefore, we eliminated PSU NF sample and did not continue to work with this polymeric sample due to its unstable fibrous morphology under ALD process of Pd decoration.

The transmission electron microscopy (TEM) images clearly reveal the decoration of $\mathrm{Pd}$ nanograins on the polymeric NF (Figure 3; Figure S3, Supporting Information). Figure 3a,b shows the monodispersed nature of Pd nanograins on the Nylon NF and does not show any appearance of aggregation. The randomly distributed $\mathrm{Pd}$ nanograins with the average size of $\approx 2 \mathrm{~nm}$ were decorated on the Nylon NF. High resolution transmission electron microscopy (HRTEM) images of individual Pd nanograins clearly evidenced the (111) facet orientation of single crystalline nanograins on the fiber surface with the lattice spacing of $0.226 \mathrm{~nm}$. The spacing difference of $0.226 \mathrm{~nm}$ along the (111) lattice of face centered cubic (fcc) Pd was confirmed by the corresponding fast Fourier transform. While using PAN NW as substrate, this recipe resulted in the similar form of monodispersed, (111) faceted nanograins, but the present density of the Pd nanograins on the PAN NF surfaces decreased (Figure 3c,d).

Since PAN NF are thicker in diameter compared to Nylon $\mathrm{NF}$, and this probably decreases the density of Pd nanograins which are located at the unit area of fiber structure. Figure S4 (Supporting Information) shows the typical energy dispersive analysis X-ray (EDAX) spectrum of Pd nanograin decorated nylon (Pd-Nylon) NF and Pd nanograin decorated PAN which exhibited the presence of Pd nanograins through the fiber matrix. Inductively coupled plasma mass spectroscopy 


\section{1st Step
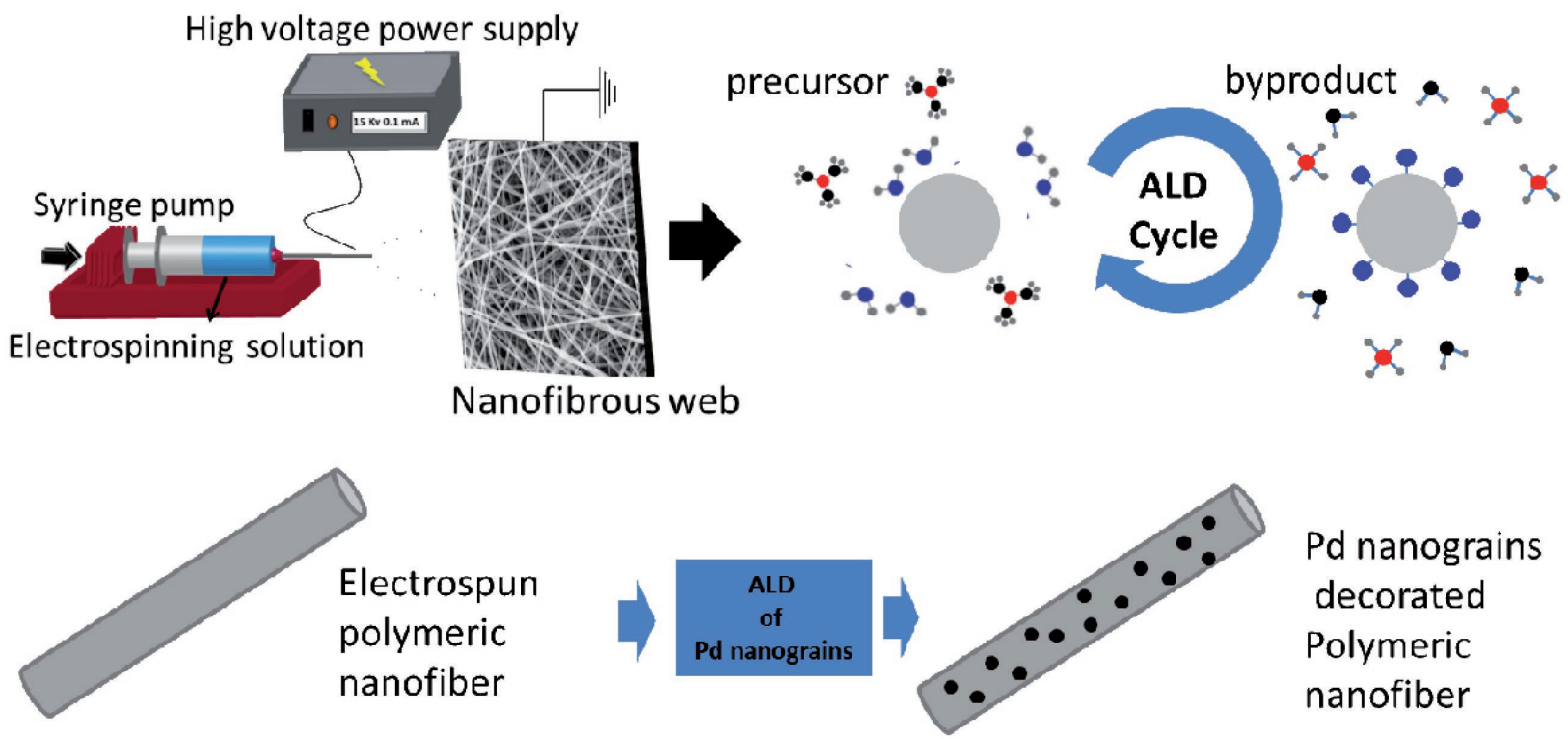

Pd nanograins decorated Polymeric nanofiber

Figure 1. Schematic representations of the processing steps (electrospinning and ALD) for the fabrication of surface-decorated metal nanostructures (Pd nanograins) onto electrospun polymeric nanofibers.

(ICP-MS) reveals the presence of Pd nanograins with the weight ratios of 26.56 and $20.32 \mu \mathrm{g} \mathrm{mg}^{-1}$ on the Nylon NW and PAN NW, respectively. The formation of Pd nanograins and their deposition density with uniform distribution onto substrate can be achieved by controlling precursor molecules in ALD process. We took this as an advantage to get low loading of Pd nanograins on the NW surface by controlling the ALD cycles and growth kinetics during the deposition process.

The surface chemical states and compositions of the $\mathrm{Pd}$ nanograin-decorated NW were investigated by X-ray photoelectron spectroscopy (XPS) (Figure 4). The survey spectra of the Pd-Nylon NF and Pd-PAN NF (Figure 4a) show the presence of $\mathrm{C}, \mathrm{N}, \mathrm{O}$, and Pd on the fiber surface, and the chemical composition was also investigated (Table S1, Supporting Information). The atomic ratio of $\mathrm{Pd}$ nanograins was estimated to be around 1.55 and $1.11 \mathrm{wt} \%$ on the Pd-Nylon NW and Pd-PAN NW. The presence of Pd on NW surface which we deduce from ICP-MS and XPS shows discrepancy, because of the quantification difference between the bulk and surface analysis. But the ratio of Pd decoration on these two different polymeric NW supports was clearly correlated with the obtained results. Figure $4 \mathrm{~b}$ shows the high-resolution Pd 3d XPS spectra of the Pd-Nylon NW and Pd-PAN NW which consist of two asymmetric peaks corresponding to $\mathrm{Pd} 3 \mathrm{~d}_{5 / 2}$ and $\mathrm{Pd} 3 \mathrm{~d}_{3 / 2}$ with prominent metallic $\mathrm{Pd}$ $\left(\mathrm{Pd}^{0}\right)$ nature. ${ }^{[26]}$ On deconvoluting the $\mathrm{Pd} 3 \mathrm{~d}$ peaks, two pairs of doublets were observed as shown in Figure 4b. On comparing the relative intensity of $\mathrm{Pd}^{0}$ and $\mathrm{Pd}^{\mathrm{II}}$ peaks in Figure $4 \mathrm{~b}$, it was noted that the most intense $\mathrm{Pd}$ exists as $\mathrm{Pd}^{0}(<60$ at \%) on both nanofibrous web surfaces which signifies the higher content of metallic nature. In addition, a higher energy shift of $0.4 \mathrm{eV}$ on Pd-PAN NW as compared with Pd-Nylon NW indicates the change in electronic states while decorating $\mathrm{Pd}$ on different polymeric surfaces. This shift may represent the interaction of $\mathrm{Pd}$ ions with the $\mathrm{C}$ or $\mathrm{O}$ groups on the polymer surface. ${ }^{[27]}$ To further understand the surface interaction and electronic inertness on the polymeric fiber surface, the substrate effect on Pd decoration was compared with the carbon NW which has similar morphology with highly conductive nature (Figure S5, Supporting Information). Due to surface interaction with the oxygen functionality, Pd exhibited multiple oxidization states in all three fiber surfaces irrespective of them being conductive or nonconductive. The multiple oxidization state may arise due to the precursor interacting on the support during the deposition process. While comparing the effect of conductive and nonconductive surfaces, Pd 3d peak has blue shifted on improving the conductive nature of the support. This shift reveals the possibility of electronic interaction of Pd on the substrate/support. In case of the two different polymeric surfaces, Pd-Nylon NW shows the blue shift when comparing with the Pd-PAN NW, which reveals the favorable possibility of surface electrical interaction on the Nylon surface than the PAN support. It may be due to possibility of carrier conduction or surface interaction extended by the deposition environment or surface functionality. The higher resolution of $\mathrm{C}$ 1s and N 1s spectra (Figure S6, Supporting Information) exhibited no significant change in the structural properties of polymeric NW after ALD deposition. Further investigating the X-ray diffraction (XRD) analysis, Pd nanograin-decorated NW do not show any notable diffraction peaks of Pd nanograins because of the very low loading of the metal and small size less than $2 \mathrm{~nm}$, which is nondetectable by the XRD (Figure S7, Supporting Information). The additional diffraction peaks at $17.3^{\circ}(100), 20.4^{\circ}$ $(100)$, and $23.0^{\circ}(010,110)$ represent the semicrystalline nature of the PAN and Nylon samples, respectively. ${ }^{[28,29]}$ The Fourier transform infrared spectrometer (FTIR) studies reveal that after 

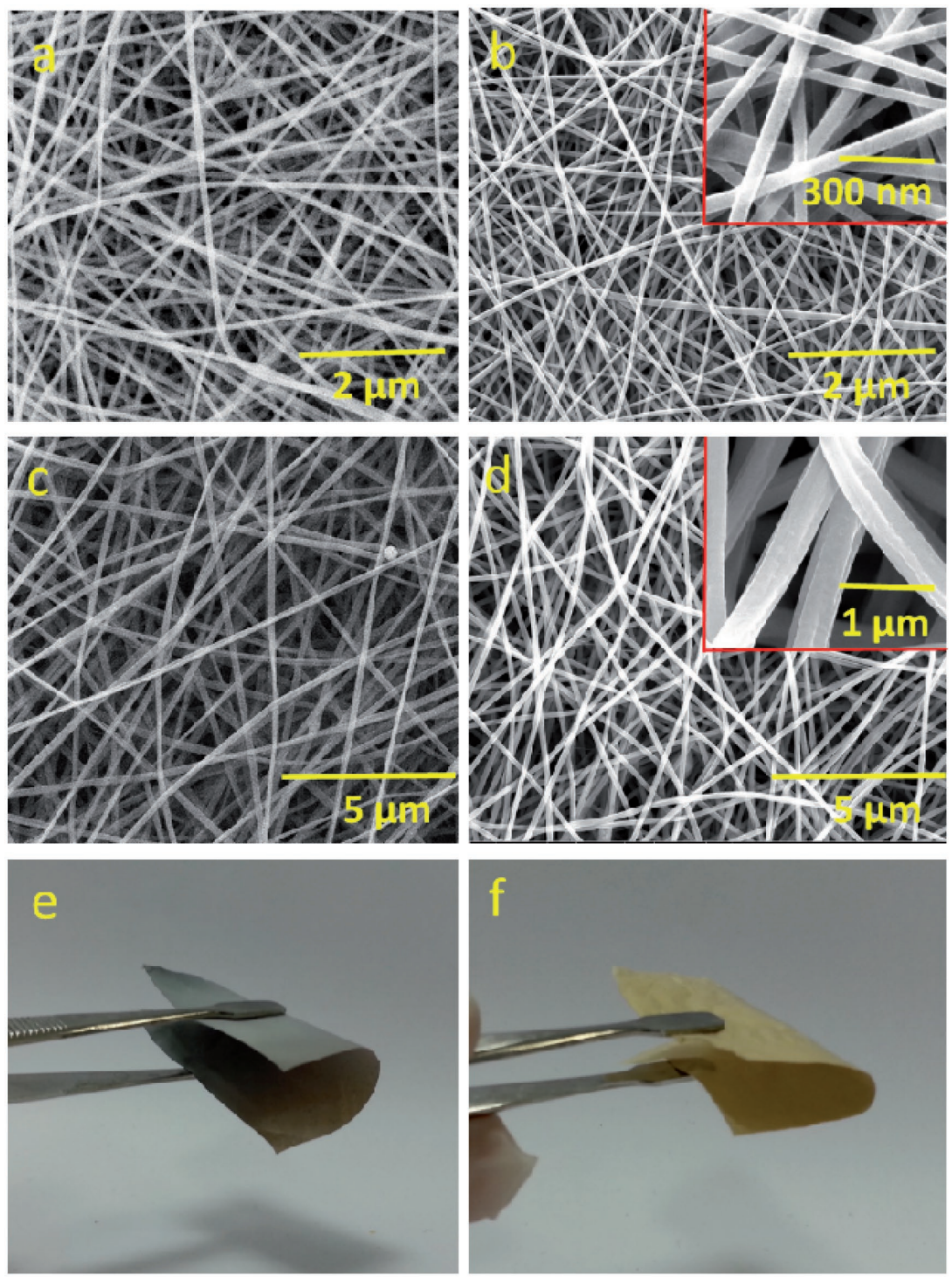

Figure 2. SEM images of a) pristine Nylon NF, b) Pd-Nylon NF, c) pristine PAN NF, and d) Pd-PAN NF. Inset shows the high magnified SEM images of the respective samples. The optical image of the flexible e) Pd-Nylon NW and f) Pd-PAN NW.

the ALD process, the chemical structure of the Nylon and PAN samples is still protected (Figure S8, Supporting Information).

$\mathrm{Pd}$ nanograin-decorated $\mathrm{NW}$ were explored for the catalytic activity toward the reduction of 4-NP by $\mathrm{NaBH}_{4}$. Colored solution of $\mathrm{NaBH}_{4}$-mixed 4-NP exhibits a strong absorption at $400 \mathrm{~nm}$ due to the 4-nitrophenolate formation. Reduction of 4-NP is kinetically restricted in the absence of catalyst on the pristine NW as shown in Figure S9 in the Supporting Information. In the presence of Pd nanograin-decorated NW, the solution starts to decolorize and time-dependent UVvis absorption spectra reveal the reduction of 4-NP to 4-AP. Additionally, Pd-PAN NW showed slightly slower catalytic rate as compared to Pd-Nylon NW (Figure 5; Figure S9, Supporting Information). This may be due to the amount of catalytic Pd loading on the NW as seen from the weight ratio from the ICP-MS results. The reduction of 4-NP to 4-AP with the catalyst follows the pseudo-first-order kinetics with the presence of excessive $\mathrm{NaBH}_{4}$. The slope from the kinetic fit determines the rate constant $(\mathrm{k})$ of the catalyst (Figure $5 \mathrm{~b}$ ). It clearly evidences that the pristine NW do not show any appealable catalytic activity to the reduction of 4-NP. Pd-Nylon NW showed $\mathrm{k}$ value of $0.0531 \mathrm{~min}^{-1}$, which was around 1.28 times faster than the Pd-PAN NW $\left(0.0413 \mathrm{~min}^{-1}\right)$, indicating higher ratio of $\mathrm{Pd}$ nanograins on the fiber surface and the presence of metallic Pd weight ratio supports the enhancement of the reduction rate. The other reason could be the higher surface interactivity due to the thinner diameter of the Nylon fibers which favor more interaction sites for the Pd nanograins for the loading on the fiber matrix and therefore provides the higher active sites during the catalytic reduction process. For comparative studies, Nylon and PAN films were also produced and decorated with Pd by ALD (Figures S10 and S11, Supporting Information). XPS results indicated that Nylon film offered much interactive nature for the Pd on its surface when compared with the PAN films, which was clearly addressed by the higher atomic wt\% of Pd on the Nylon surface (Figure S12, Supporting Information). When polymeric films are compared with each other, Pd-Nylon film has shown better catalytic activity than Pd-PAN film possibly due to higher loading of Pd on the surface. When comparing the catalytic performance of Pd-decorated polymeric NW and films, the NW provided more interactive sites for Pd to interact with the substrate surface which increases the loading density and uniform distribution of Pd and therefore offers higher catalytic efficiency (Figure 5b). In contrast to film form, NW form of polymeric substrates offers higher flexibility with high specific surface area for the Pd decoration. The specific surface area of the pristine PAN NW and Nylon NW was obtained as 6.591 and $40.311 \mathrm{~m}^{2} \mathrm{~g}^{-1}$, respectively. When decorated with the Pd nanograins by ALD, surface area was increased to 12.273 and $54.157 \mathrm{~m}^{2} \mathrm{~g}^{-1}$, respectively, for the Pd-PAN NW and PdNylon NW samples (Figure S13, Supporting Information). This clearly reveals that the Nylon NW offers higher surface area to improve the loading capacity of Pd which results in higher catalytic efficiency. Deposited Pd on the fiber surface or the deposition process itself additionally induces the surface modification, which improvises the surface area of the polymeric NW. Comparing with the commercially available platinum on graphitized carbon $(\mathrm{Pt} / \mathrm{C})$ catalyst, Pd-decorated polymeric NW and films exhibited slower catalytic performance, which may be due to the higher wt\% of metal ion (20 wt\%) concentration in $\mathrm{Pt} / \mathrm{C}$ catalyst, which is nearly ten times higher than our Pd-loaded NW samples. 

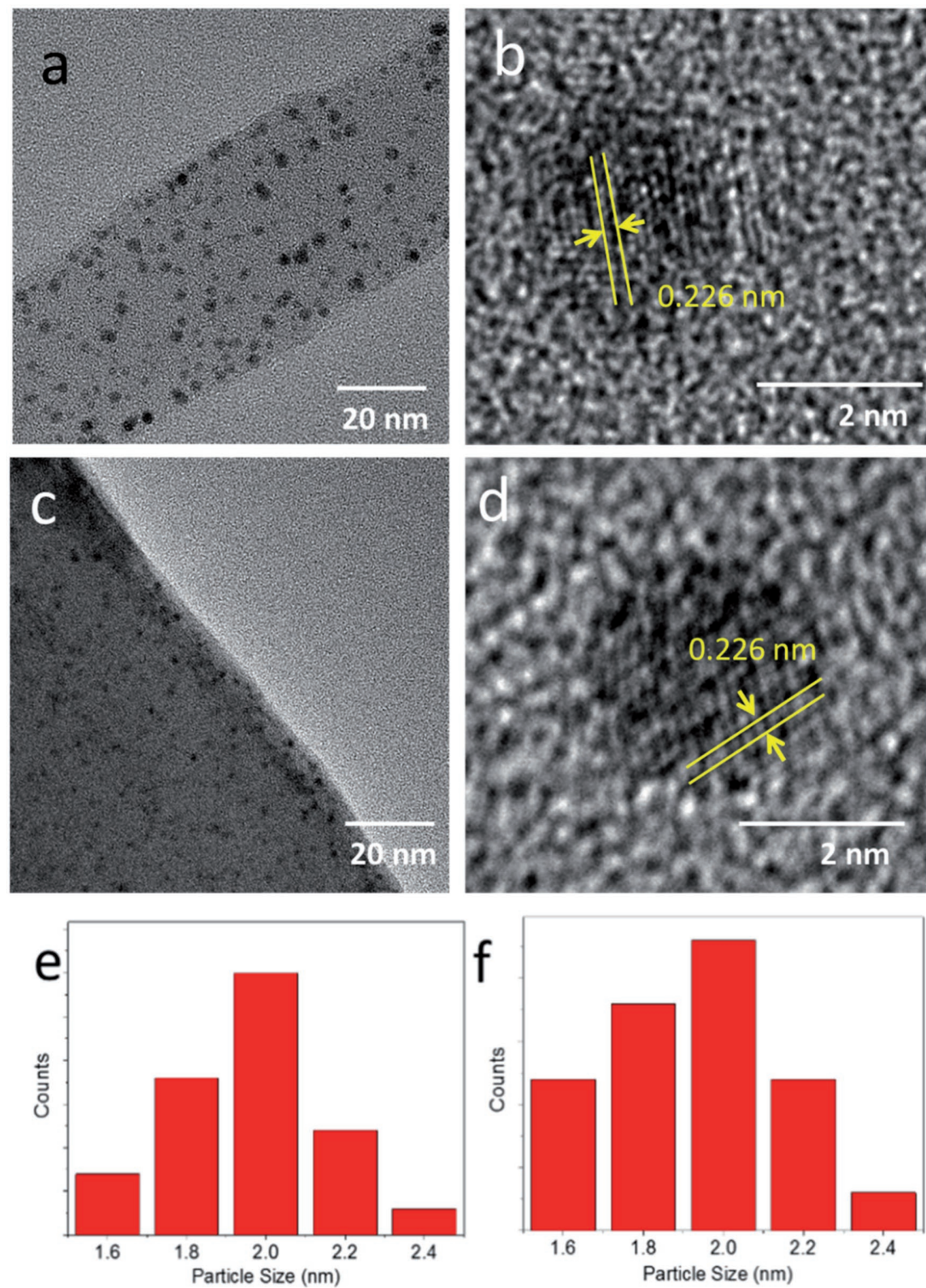

Figure 3. TEM and HRTEM images of a,b) Pd-Nylon NF and c,d) Pd-PAN NF. Particle size distribution of Pd nanograins on e) Nylon NF and f) PAN NF surface.

$\mathrm{Xu}$ et al. ${ }^{[20]}$ have predicted (111) facet of Pd ions to be the highly interactive site with the effective charge distribution, which was calculated by the density functional theory. The exposure of (111) facets on the polymeric surface imparts the additional advantage of high activity and sensitivity for the reduction of 4-NP. On analyzing the different dosage of catalytic Pd-Nylon NW (Figure 5c), kinetic rate was seen to have increased with linear increase of the $\mathrm{k}$ value on increasing the catalytic dosage (represented by the weight ratio of the Pd-Nylon NW). The activation energy $\left(E_{a}\right)$ of the catalytic performance was calculated through the temperature-dependent catalytic reaction rate on the Pd-Nylon NW. Figure $5 d$ reveals the kinetic plot of Pd-Nylon NW at various temperatures from 12 to $42{ }^{\circ} \mathrm{C}$. The exhibition of the linear relationship with the $\operatorname{In}\left(A_{\mathrm{t}} / A_{0}\right)$ versus time $(t)$ favors the Arrhenius-type dependence on varying the reaction temperature. The calculated $E_{\mathrm{a}}\left(1.705 \mathrm{~J} \mathrm{~mol}^{-1}\right)$ from the plot reveals the favorable surface catalytic reaction which significantly enhances lower energy barrier of the 4-NP reduction. To understand the mechanism behind the catalytic process as illustrated from Figure 6, the reduction of 4-NP caused by the $\mathrm{BH}_{4}^{-}$ion by the rapid hydrogen transfer and effective adsorption of the reactant has to be comprehended. ${ }^{[30]}$ Initially, Pd ions effectively interacted with the fiber surface and initiated the electron transfer to the fiber's surface and modified the electronic states of the Pd ions. This initiated the favorable electrophilic behavior on the metal ions to interact with or adsorb the 4-nitrophenolate and $\mathrm{BH}_{4}$ ions, which facilitated the hydrogenation process on the catalytic surface. ${ }^{[31]}$ This can be ascribed as Langmuir-Hinshelwood mechanism, where both reactants need to be adsorbed on the catalyst prior to reaction. ${ }^{[32]}$ Further, electrodeficient Pd ions get electrons from the $\mathrm{BH}_{4}$ ions and favor the generation of hydrogen atom from the fractionation of $\mathrm{B}-\mathrm{H}$ bond. The thermodynamically unstable nature of the active hydrogen atoms favorably reacted with the 4-nitrophenolate ions through the conventional hydrogenation process. Figure $5 \mathrm{~d}$ reveals the four consecutive catalytic cyclic performance of the Pd-Nylon NW. Results show that after three cycles, the catalytic activity starts deterioration because of the decrease in the metallic nature and the leaching of $\mathrm{Pd}$ ions during every recycle process. Note that the metallic content of $\mathrm{Pd}$ ions $\left(\mathrm{Pd}^{0}\right.$ state) was reduced below $40 \%$ during the cyclic process which has been confirmed from the XPS spectra (Table S2, Supporting Information), and therefore reduces the catalytic activity during the multiple cyclic catalytic process. Yet, the morphology of the Pd-decorated NW samples was stable and kept its fiber morphology after catalytic process which has been confirmed from the SEM images (Figure S14, Supporting Information). Further, the life test of the metaldecorated polymeric NW was investigated to understand the catalytic performance with the function of time span reactivity in ambient condition. As compared with catalytic activity of Pd-decorated polymeric NW, the older ones (prepared 6 and 12 months before) exhibited slower catalytic rate of $52 \%$ and $74 \%$ of the freshly prepare one. The loss in catalytic efficiency on the older fibers may be due to the surface oxidization of the Pd metal ions in ambient condition related with the function of time span. The Pd-decorated polymeric NW samples were highly stable in the pollutant environment, even after immersion for a long time. Figure S15 

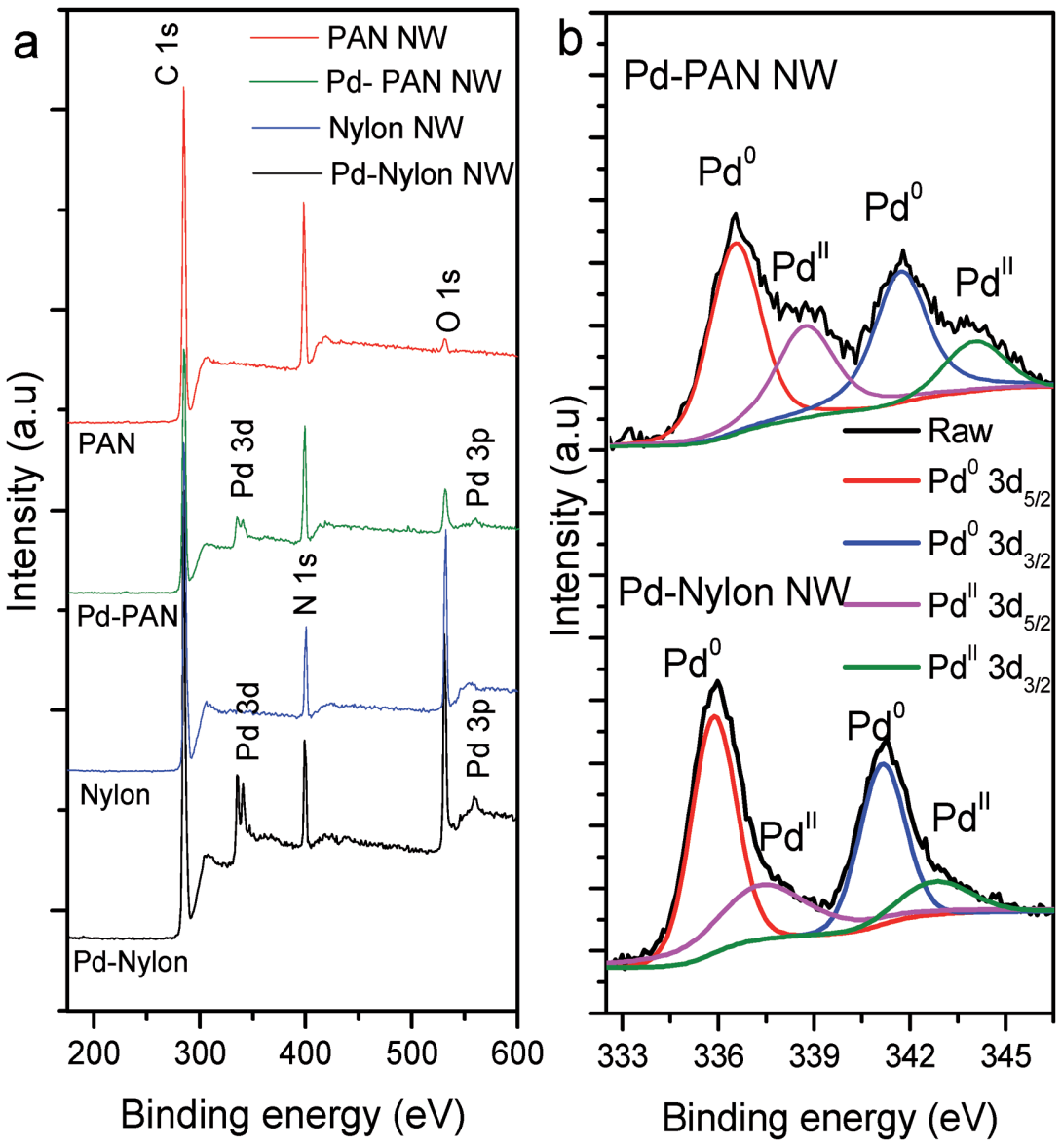

Figure 4. XPS spectra of Pd nanograin-decorated nanofibrous webs a) survey spectrum and b) high-resolution Pd-3d spectrum, respectively.

(Supporting Information) shows the SEM images of the Pd-decorated polymeric web immersed for nearly 2 weeks in 4-NP/4-AP solution which exhibited stable structural nature.

\section{Conclusion}

In summary, we have fabricated Pd nanograin-decorated flexible catalytic polymeric nanofibrous membranes for the reduction of 4-NP by combining the electrospinning and ALD techniques. Using the electrospun polymeric NW as a growth substrate in the ALD, it initiated Pd to be in the monodispersive nature with the single crystalline facet oriented on the nanofiber surface. The low trace of Pd-supported polymeric NW were utilized for the effective catalytic application for the reduction of 4-NP to 4-AP by $\mathrm{NaBH}_{4}$. The catalytic activity of the Pd-decorated polymeric NW was compared with the Pd-decorated polymeric films, and it results in that the polymeric NW would be perfect platform of higher density of Pd intergradation with flexible free standing finish with higher specific surface area. While comparing the two different polymeric NW support, Nylon NW were provided higher specific surface area and much preferable surface interaction for loading of higher density of monodispersive Pd nanograins, which initiates the higher catalytic performance than PAN NW. The conformal decoration of $\mathrm{Pd}$ on the polymeric nanofiber surface favored the electron transfer and improved the adsorption of negatively charged reactant and initiated the hydrogen transfer to boost the reduction of nitro component. Easy recoverable nature with the reusability was interested to move further on the catalyst over the flexible and free-standing polymeric nanofibrous membranes.

\section{Experimental Section}

Materials: Polyacrylonitrile (PAN, $M_{w} \approx 150000$, Scientific Polymer Products, Inc.), Nylon $66\left(M_{w}\right.$ 230 000-2 800 000, Scientific Polymer Products, Inc.), Polysulfone (PSU, Scientific Polymer Products, Inc.), palladium(II) hexafluoroacetylacetonate ( $\mathrm{Pd}(\mathrm{hfac})_{2}$, Strem Chemicals), formalin $(\mathrm{HCOH}$, Sigma-Aldrich), N,N-dimethylformamide (DMF, Pestanal, Riedel), formic acid $(\mathrm{HCOOH}$, $98 \%$, Sigma-Aldrich), platinum on graphitized carbon (Pt/C, 20 wt\%, Sigma-Aldrich), N,Ndimethylacetamide (DMAc, 99\%, Sigma-Aldrich), acetone (>99\%, Sigma-Aldrich), 4-nitro-phenol (4-NP, 99\%, Alfa Aesar), methanol $\left(\mathrm{CH}_{3} \mathrm{OH}\right.$, Sigma-Aldrich), and sodium borohydride $\left(\mathrm{NaBH}_{4}\right.$, fine granular, Merck) were obtained commercially. All materials were used without any purification. Deionized (DI) water was obtained from the Millipore Milli-Q system.

Electrospinning: For electrospun uniform and bead-free nanofibers, clear solutions of Nylon, PAN, and PSU in formic acid, DMF, and DMAc at $8 \%, 13 \%$, and $32 \%(\mathrm{w} / \mathrm{v}$, with respect to solvent) polymer concentrations, respectively, were prepared separately. After that, the homogeneous solutions of each polymer were loaded in 3 or $10 \mathrm{~mL}$ syringe fitted with a metallic needle of $0.4 \mathrm{~mm}$ inner diameter. The syringe was located horizontally on the syringe pump (KD Scientific, KDS 101) with a $0.5-1 \mathrm{~mL} \mathrm{~h}^{-1}$ flow rate. A high voltage of 10-15 kV was applied by high voltage power supply (Matsusada, AU Series) to the tip of the needle to initiate the electrospinning jet movement through the stationary plate metal collector which was covered with aluminum foil and positioned at $10 \mathrm{~cm}$ from the end of the tip. The electrospinning process was carried out at $\approx 25{ }^{\circ} \mathrm{C}$ and $22 \%$ relative humidity in an enclosed Plexi-glass chamber. All the collected nanofibrous webs were placed in the hood for overnight to remove residual solvent if any present.

Surface Decoration of Pd Nanograins on Nanofibrous Webs by ALD: ALD was employed to free-standing electrospun NW for the decoration of Pd nanograins on the surface of nanofibers. Pd nanograins were decorated directly onto $\mathrm{NW}$ by using $\mathrm{Pd}(\mathrm{hfac})_{2}$ as $\mathrm{Pd}$ precursor and formalin $(\mathrm{HCOH}, 30 \%$ in aqueous solution with $15 \%$ methanol) as counter reactant. The temperature of $\mathrm{Pd}$ precursor was held at $70^{\circ} \mathrm{C}$ to obtain a proper vapor pressure. $\mathrm{N}_{2}$ was used as carrier gas flow rate of $20 \mathrm{sccm}$ under dynamic vacuum condition. Fifteen cycles were deposited for the decoration of Pd nanograins at $200^{\circ} \mathrm{C}$.

Characterizations: The morphology of the pristine and Pd nanograindecorated NW was investigated by using SEM (FEI-Quanta 200 FEG). The NW was coated with $5 \mathrm{~nm}$ Au/Pd for SEM imaging. Around 100 fiber diameters were measured from the SEM image of pristine nanofibers in order to calculate the average fiber diameter. Detailed morphological investigation of the Pd-PAN and Pd-Nylon nanofibers was performed by using TEM (FEI-Tecnai G2F30, Hillsboro). Small pieces of Pd nanograindecorated NW were sonicated in ethanol for $5 \mathrm{~min}$ to obtain individual 

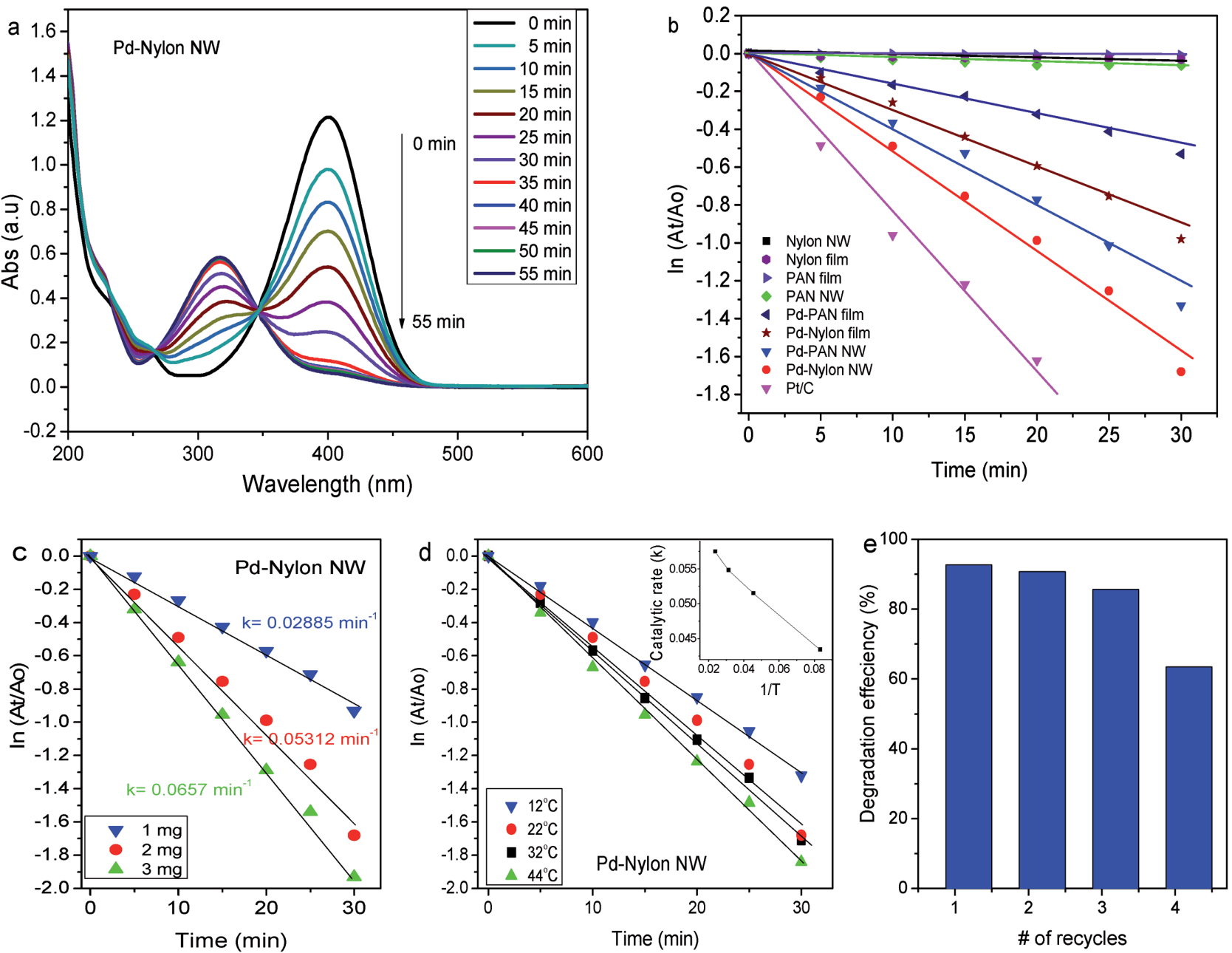

Figure 5. The typical time-dependent UV-vis absorption spectra for the reduction of 4-NP over Pd-Nylon nanofibrous web. b) Plots of $\ln \left(A_{t} / A_{0}\right)$ versus reaction time for the reduction of 4-NP by catalytic samples (pristine nanofibrous webs and films, Pd-decorated polymeric nanofibrous webs and films, commercial Pt/C sample). The reaction parameters: $[4-\mathrm{NP}]=0.01 \times 10^{-3} \mathrm{M},\left[\mathrm{NaBH}_{4}\right]=7 \times 10^{-3} \mathrm{M}$, the amount of catalysis is $2.0 \mathrm{mg}$ in $3 \mathrm{~mL}$. c) Influence of catalyst dosage of Pd-Nylon nanofibrous web. d) Influence of temperatures on the kinetic constant $k$ over the resulting Pd-Nylon nanofibrous web. Inset indicates the corresponding Arrhenius curve. e) Catalytic activity of Pd-Nylon nanofibrous web in consecutive cycles.

nanofibers dispersed through the solvent, followed by dropping the suspensions onto the copper TEM grids ( $\mathrm{HC200})$, and were allowed to dry under IR lamp for a few minutes prior to TEM imaging. Scanning transmission electro microscopy (STEM) spectrum was recorded with an energy-dispersive X-ray spectroscopy (EDX) attached to HRTEM. XPS measurements were carried out by a Thermo Scientific spectrometer with an $\mathrm{Al} \mathrm{K \alpha} \mathrm{X}$-ray source for detecting the surface-elemental composition of pristine and Pd nanograin-decorated NW. XPS data were taken from $400 \mu \mathrm{m}$ diameter circular spot on the sample surface by means of a flood gun charge neutralizer system equipped with a monochromated $\mathrm{Al} \mathrm{K} \alpha$ $\mathrm{X}$-ray source $(h v=1486.6 \mathrm{eV})$. Wide energy survey scans were obtained over a 0-1360 eV binding energy range, at pass energy of $150 \mathrm{eV}$, and with an energy step of $1 \mathrm{eV}$. Pd 3d high-resolution XPS scan was also taken at pass energy of $30 \mathrm{eV}$, and with energy steps of $0.1 \mathrm{eV}$ in order to analyze the bonding states. The powder XRD patterns were recorded by using a PANalytical X'Pert Multi-Purpose X-ray Diffractometer with a $\mathrm{Cu} \mathrm{K} \alpha$ radiation source $(\lambda=0.15406 \mathrm{~nm})$. FTIR (Bruker-VERTEX 70) was used to record the infrared spectra of nanofibrous webs. For measurement, the samples were mixed with potassium bromide $(\mathrm{KBr})$ and pressed as pellets. The scans (64 scans) were recorded between 4000 and $400 \mathrm{~cm}^{-1}$ at a resolution of $4 \mathrm{~cm}^{-1}$. The bulk compositions were analyzed using a Thermo, $X$ series II ICP-MS. For the ICP-MS measurement, $1 \mathrm{mg}$ of NW was completely dissolved in 1:1 ratio of nitric acid: hydrochloric acid $(\mathrm{HCl})$ $(2 \mathrm{~mL})$ and diluted further in $2 \% \mathrm{HCl}$ for the ICP-MS measurement. Pd standard dissolved in $\mathrm{HCl}$ was prepared with different concentration (250, $125,62.5,32.25$, and $16.125 \mathrm{ppb}$ ) as a known measurement, and through this, the amount of Pd in the NW was quantified.

Catalytic Reduction of 4-NP: The catalytic performance of Pd nanograindecorated NW samples was investigated by the reduction of 4-NP to 4-AP along with the existence of $\mathrm{NaBH}_{4}$. Typically, $3 \mathrm{~mL}$ of 4-NP $(0.1 \times$ $10^{-3} \mathrm{M}$ ) was introduced to a standard quartz cuvette with $1 \mathrm{~cm}$ path length and followed by adding $3 \mathrm{~mL}$ of $\mathrm{NaBH}_{4}\left(7 \times 10^{-3} \mathrm{M}\right)$. The initial concentration ratio of $\mathrm{NaBH}_{4}$ to 4-NP was fixed to be equal. Note that $2 \mathrm{mg}$ worth of Pd nanograin-decorated NW (approximately equivalent to $20 \mu \mathrm{g} \mathrm{mg}^{-1}$ of Pd metal ions) was added to $5 \mathrm{~mL}$ of the reaction solution and in this blend solution and shaken at $320 \mathrm{rpm}$. The yellow solution gradually faded as the reaction proceeded. Meanwhile, the catalytic reaction was monitored in the progressing time at $400 \mathrm{~nm}$ by using UVvis spectrophotometer (Varian, Carry 100). For comparison, the same experiment was also performed for the pristine nanofibrous web. In terms of analyzing the catalytic performance of Pd nanograins respectively with the dosage, different weight ratios (1, 2, 3, and $4 \mathrm{mg}$ ) of Pd-decorated NW were exposed to same experiment procedure. In order to analyze the thermocatalytic properties, same experiment procedure was done in 

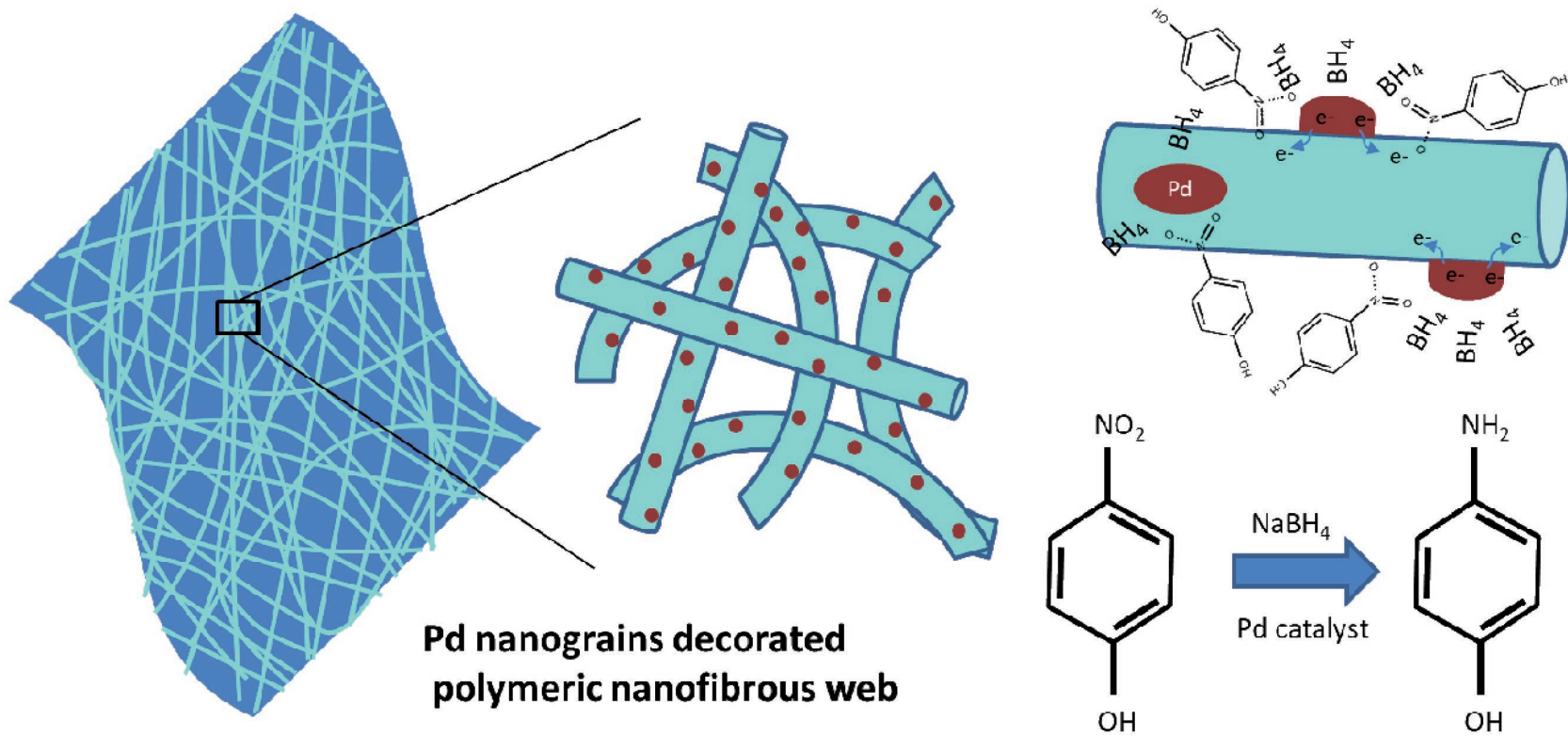

Figure 6. Schematic illustration of the synergistic catalytic mechanism for the 4-NP reduction over Pd nanograin-decorated NW.

different temperature $\left(12-42^{\circ} \mathrm{C}\right)$, and the activation energy was calculated from the kinetic fit. Finally, the reusability performance of Pd-Nylon NW was checked by applying a slight washing step to the NW in water after each catalyst reaction, and then NW were dried and used for the next cycles. After reusability test, the morphology of NW was imaged by SEM.

\section{Supporting Information}

Supporting Information is available from the Wiley Online Library or from the author.

\section{Acknowledgements}

K.S.R. and A.C. contributed equally to this work. The Scientific and Technological Research Council of Turkey (TUBITAK, project \#115Z488) was acknowledged for funding this research. The authors thank Zehra Irem Yildiz for performing FTIR measurements.

\section{Conflict of Interest}

The authors declare no conflict of interest.

\section{Keywords}

atomic layer deposition, electrospinning, metal catalyst, Pd nanograins, polymeric nanofibers

Received: June 3, 2017 Revised: August 15, 2017 Published online: October 16, 2017

[1] A. Serrà, X. Alcobé, J. Sort, J. Nogués, E. Vallés, J. Mater. Chem. A 2016, 4, 15676.
[2] C. K. Wang, S. T. Huang, J. G. Lin, Water Sci. Technol. 2000, 42, 155.

[3] M. A. Oturan, J. Peiroten, P. Chartrin, A. J. Acher, Environ. Sci. Technol. 2000, 34, 3474.

[4] M. Q. Yang, B. Weng, Y. J. Xu, J. Mater. Chem. A 2014, 2, 1710.

[5] P. Cañizares, M. Carmona, J. Lobato, F. Martínez, M. A. Rodrigo, Ind. Eng. Chem. Res. 2005, 44, 4178.

[6] J. A. Johnson, J. J. Makis, K. A. Marvin, S. E. Rodenbusch, K. J. Stevenson, J. Phys. Chem. C 2013, 117, 22644.

[7] B. R. Cuenya, Acc. Chem. Res. 2013, 46, 1682.

[8] S. Gu, S. Wunder, Y. Lu, M. Ballauff, R. Fenger, K. Rademann, B. Jaquet, A. Zaccone, J. Phys. Chem. C 2014, 118, 18618.

[9] W. Zang, G. Li, L. Wang, X. Zhang, Catal. Sci. Technol. 2015, 5, 2532.

[10] M. Imran, A. B. Yousaf, X. Zhou, Y. F. Jiang, C. Z. Yuan, A. Zeb, N. Jiang, A. W. Xu, J. Phys. Chem. C 2017, 121, 1162.

[11] X. Wang, H. Shi, J. H. Kwak, J. Szanyi, ACS Catalysis 2015, 5, 6337.

[12] Y. Imura, K. Tsujimoto, C. Morita, T. Kawai, Langmuir 2014, 30, 5026.

[13] J. Zhen, D. Liu, X. Wang, J. Li, F. Wang, Y. Wang, H. L. Zhang, Dalton Trans. 2015, 44, 2425.

[14] G. Q. Gao, L. Lin, C. M. Fan, Q. Zhu, R. X. Wang, A. W. Xu, J. Mater. Chem. A 2013, 1, 12206.

[15] A. Abedini, A. A. A. Bakar, F. P. Larki, S. Menon, M. S. Islam, S. Shaari, Nanoscale Res. Lett. 2016, 11, 287.

[16] A. D. Banadaki, A. Kajbafvala, J. Nanomater. 2014, 2014, 985948.

[17] Z. N. Xu, J. Sun, C. S. Lin, X. M. Jiang, Q. S. Chen, S. Y. Peng, M. S. Wang, G. C. Guo, ACS Catalysis 2013, 3, 118.

[18] R. Narayanan, M. A. El-Sayed, J. Am. Chem. Soc. 2004, 126, 7194

[19] R. Narayanan, M. A. El-Sayed, J. Phys. Chem. B 2004, 108, 5726.

[20] R. Narayanan, M. A. El-Sayed, Nano Lett. 2004, 4, 1343.

[21] R. Wang, H. He, L. C. Liu, H. X. Dai, Z. Zhao, Catal. Sci. Technol. 2012, 2, 575

[22] A. X. Yin, X. Q. Min, Y. W. Zhang, C. H. Yan, J. Am. Chem. Soc. 2011, 133, 3816.

[23] P. Serp, B. Machado, RSC Catal. Ser. 2015, 23, 1.

[24] L. Zhang, X. Gong, Y. Bao, Y. Zhao, M. Xi, C. Jiang, H. Fong, Langmuir 2012, 28, 14433

[25] M. Zhang, X. Zhao, G. Zhang, G. Wei, Z. Su, J. Mater. Chem. B 2017, 5, 1699. 
[26] H. Xu, L. X. Ding, C. L. Liang, Ye. X. Tong, G. Ren, NPG Asia Mater. 2013, 5, e69.

[27] P. Stoyanov, S. Akhter, J. M. White, Surf. Interface Anal. 1990, 15, 509.

[28] C. O. Akgun, F. Kayaci, S. Vempati, A. Haider, A. Celebioglu, E. Goldenberg, S. Kizir, T. Uyar, N. Biyikli, J. Mater. Chem. C 2015, 3, 5199 .
[29] M. Makaremi, C. L. Lim, P. Pasbakhsh, S. M. Lee, K. L. Goh, H. Chang, E. S. Chan, RSC Adv. 2016, 6, 53882.

[30] L. Liu, R. Chen, W. Liu, J. Wu, D. Gao, J. Hazard. Mater. 2016, 320, 96.

[31] Z. D. Pozun, S. E. Rodenbusch, E. Keller, K. Tran, W. J. Tang, K. J. Stevenson, G. Henkelman, J. Phys. Chem. C 2013, 117, 7598.

[32] F. H. Lin, R. A. Doong, Appl. Catal., A 2014, 486, 32. 